\title{
Redundant Neurovascular Imaging: Who Is to Blame and What Is the Value?
}

\author{
(D)E. Beheshtian, (DS. Emamzadehfard, (D) S. Sahraian, (D). Jalilianhasanpour, and (DD.M. Yousem
}

\begin{abstract}
BACKGROUND AND PURPOSE: Excessive use of neurovascular imaging studies such as Doppler ultrasound, CTA, MRA, and DSA adds cost to the evaluation of patients with new neurologic deficits. We sought to determine to what extent redundant neurovascular imaging is generated by radiologists' recommendations and the agreement rates among modalities in this setting.
\end{abstract}

MATERIALS AND METHODS: The radiology reports of 300 consecutive patients admitted for acute stroke to determine the frequency of the following: 1) >1 neurovascular study performed, 2) recommendation for another study, 3) recommendation made by the radiologist, and 4) agreement rates among these redundant neurovascular imaging studies.

RESULTS: Among the 300 consecutive patients, 125 had redundant neurovascular imaging, accounting for 144 redundant studies. These included 75/125 redundant neurovascular imaging studies after MRA, 48/125 after CTA, and 2/125 after Doppler ultrasound. The radiologist recommended another vascular study in $22 / 125$ (17.6\%) patients; the rest of the recommendations were made by clinicians. The second study agreed with the first in 54.6\% (12/22) of cases recommended by radiologists and 73.8\% (76/103) recommended by clinicians $(P$ value $=.06$ ). CTA agreed with MRA, carotid Doppler ultrasound, and DSA in $66.7 \%, 66.7 \%$, and $55.6 \%$, respectively. MRA agreed with Doppler ultrasound and DSA in $78.3 \%$ and $66.7 \%$, respectively.

CONCLUSIONS: Of cases with redundant neurovascular imaging, most were generated by clinicians, but radiologists recommended redundant neurovascular imaging in $17.6 \%$ of patients; $81.8 \%$ occurred following MRA. Overall, most secondary studies (68.8\%) confirmed the findings of the first study. Such low-value, same-result redundant neurovascular imaging was more common when clinicians ordered the studies $(73.8 \%)$ than when radiologists ordered them $(54.6 \%)$.

ABBREVIATIONS: DUS = Doppler ultrasound; RNI = redundant neurovascular imaging

$\mathrm{T}$ he diagnostic work-up and clinical decision-making in the setting of acute stroke depends on the use of accurate imaging modalities. Noninvasive vascular imaging is recommended in the American College of Radiology Practice Guideline for new neurologic deficits, and multiple choices of modalities are available, including CTA, MRA, and Doppler ultrasound (DUS). In the clinical setting in which the initial noninvasive test is nondiagnostic or inconclusive, a second noninvasive test may be corroborative or provide more definitive information. Such

Received September 6, 2019; accepted after revision October 2.

From the Russell H. Morgan Department of Radiology and Radiological Science (E.B., S.S., R.J., D.M.Y.), Johns Hopkins Medical Institution, Baltimore, Maryland; and Department of Radiology (S.E.), University of Texas Health Science Center, San Antonio, Texas.

E. Beheshtian and S. Emamzadehfard are co-first authors with equal contributions. Please address correspondence to David M. Yousem, MD, MBA, $600 \mathrm{~N}$ Wolfe St, Phipps B1100F, Baltimore, MD 21287; e-mail: dyousem1@jhu.edu; @dyouseml; @EBeheshtian

http://dx.doi.org/10.3174/ajnr.A6329 noninvasive studies may serve as screening examinations before or instead of a definitive invasive test such as DSA. ${ }^{1-3}$

However, for most cooperative patients, a single noninvasive neurovascular imaging test should be sufficient to determine future risk of stroke based on the degree of vascular stenosis in the neck or head. This is predicated, in part, on the NASCET study which, to this day, remains the basis for assessing vascular stenosis for surgical intervention. ${ }^{4}$ Recently, a study that compared potential drivers of health care spending in the United States with those of 10 of the highest income countries showed that the United States spends approximately twice as much as other nations despite comparable numbers of hospital beds (2.8 per 1000). The United States had the second highest use of MR imaging (118 per 1000) and the third highest CT use (245 per $1000)$ versus other countries. ${ }^{5}$

The performance of multiple redundant imaging modalities is potentially a low-yield, high-cost endeavor and results in transfer delay, unnecessary morbidity, and increased resource use. ${ }^{6}$ Recent studies have shown that, from 1994 through 2015, neuro- 
Table 1: Baseline characteristics of patients with stroke

\begin{tabular}{lc}
\hline Characteristics & \\
\hline Sex (No.) (\%) & $162 / 300(54)$ \\
Male & $138 / 300(46)$ \\
Female & $63.3 \pm 13.9$ \\
Age (mean) (yr) & \\
Ethnicity (No.) (\%) & $198 / 300(66)$ \\
African American & $65 / 300(21.7)$ \\
White & $37 / 300(12.3)$ \\
Other & $248 / 300(82.7)$ \\
MRA (No.) (\%) & $146 / 300(48.7)$ \\
CTA (No.) (\%) & $15 / 300(5.0)$ \\
Diagnostic DSA (No.) (\%) & $34 / 300(11.3)$ \\
DUS (No.) (\%)
\end{tabular}

imaging use rates per 1000 emergency department visits increased 660\% overall. From 2001 to 2015, rates increased $14,600 \%$ and $17,781 \%$ for head and neck CTA, respectively, and $525 \%$ and $667 \%$ for head and neck MRA, respectively. ${ }^{7}$ Increasingly, patients with stroke are undergoing $\geq 2$ neuroimaging (CT and MR imaging) and neurovascular (CTA, MRA, DUS, and DSA unrelated to intravascular treatment) studies. Although some patients certainly merit careful evaluation, unnecessary duplicative vascular imaging observed in patients with stroke/TIA may lead to high-cost, low-value care. ${ }^{8}$

Such redundancy may result in between-study discrepancies, forcing yet a third imaging technique to adjudicate the 2, thereby potentially further increasing radiation, contrast exposure, falsepositives, cost, and inconvenience. This compounds the wasteful health care expense and also leads to inefficiencies for patients and health care providers. In the setting of stroke, radiologists often blame the clinical service for ordering redundant neurovascular studies. Radiologists fail to recognize that their nondefinitive interpretations of reports may lead to additional testing or that they themselves recommend corroborative examinations that lead to added costs. We sought to assess the incidence of redundant neurovascular imaging (RNI) studies and determine the sources and referrers of such repetitive imaging, the reason for these duplicative examinations, the agreement rate when performing $>1$ imaging study on the same vascular anatomy, and guidance to reduce unnecessary repetitive imaging.

We hypothesized the following: 1) Such redundancy would occur in $<10 \%$ of cases, 2 ) the clinical services (as opposed to radiologists) would be responsible for $>90 \%$ of these cases, and 3) that MRA and CTA findings would be interchangeable. Therefore, ordering both studies would have low added value.

\section{MATERIALS AND METHODS}

This Health Insurance Portability and Accountability Act-compliant study was approved by the institutional review board at Johns Hopkins Medical Institution (approval No. 00102719).

This retrospective study was conducted using a maintained data base of patients with new-onset stroke admitted to our tertiary care academic medical institution. Informed consent requirements were waived due to the retrospective nature of the study. The data base was queried to identify 300 consecutive patients who presented to the emergency department and were subsequently admitted to the neurology stroke service for the 30 months between
February 2015 and August 2017. Only redundant studies from the same hospital admission/emergency department visit were included. Patients transferred from nearby community hospitals were not included to allow accurate inferences to be made about imaging ordering patterns, and patients who underwent interventional procedures such as thrombectomy were excluded from study.

The patients' electronic health records (Epic; 1979 Milky Way, Verona, Wisconsin) were reviewed to acquire demographics, risk factors, and different types of neuroimaging. Since the incidence of vascular stenosis is different based on their race/ethnicity, the ethnicity of patients extracted from their medical records. We specifically queried regarding incidences of carotid DUS, CTA, diagnostic DSA, and MRA occurring within the same admission. We looked at the individual official reports to identify recommendations and to ascertain whether the recommendation wording included the character string "recommend" such as "Consider CTA/MRA" or "Further evaluation with CTA/MRA, if clinically indicated." The reports were reviewed to determine the following: 1) the frequency of redundant studies, 2) the reasons specified for ordering the second neurovascular study, and 3) the rate at which radiologists recommended the additional studies in the reports.

We used the NASCET criteria to measure vascular stenoses in the neck and measured intracranial disease based on the narrowest segment diameter versus the closest normal segment diameters using electronic calipers. When studies showed $>50 \%$ stenosis in any vessel in the neck or brain, the findings were positive. This was determined at a study level, not individual vessels, because the treatment outcome for the stenoses in the neck or brain was likely the same whether $\geq 1$ vessel showed atherosclerotic narrowing. The $50 \%$ value is the threshold at our institution for the initiation of antiplatelet drugs and/or surgical consideration (>70\% stenosis). We used the TOAST (Trial of Org 10172 in Acute Stroke Treatment) criteria to classify stroke etiology. This was based on the stroke neurology team assessment and final imaging findings. ${ }^{9}$

Statistical analyses were conducted using STATA software (Version 12; StataCorp, College Station, Texas).

\section{RESULTS}

The baseline characteristics of patients with and without RNI and studies performed are reported in Table 1.

Of the 300 patients admitted to our institution for ischemic stroke during February 2015 to August 2017, one hundred twenty-five $(41.7 \%)$ had multiple vascular studies ordered, and among these 125 patients, 144 duplicative neurovascular studies were performed. On average, patients with stroke had a mean of 1.5 neurovascular imaging studies during the admission/emergency department visit. The time lapse between studies in patients with duplicative imaging is reported in the Figure.

In $17.6 \%(22 / 125)$ of patients with redundant imaging, the RNI was performed on the basis of a recommendation by the radiologist placed in the impression section of the report $(7.3 \%$ of total patients with stroke). When radiologists recommended additional radiologic studies, 18/22 (81.8\%) occurred following an MRA and 4/22 (18.2\%) occurred after CTA. On the other hand, clinicians ordered additional imaging following MRA in 57 cases and after CTA in 44 cases $(P$ value $=.027)$. 
Of the instances in which the radiologist recommended a second study, that second study confirmed the first study in $54.6 \%(12 / 22)$ of cases and disagreed with the first study in $45.4 \%(10 / 22)$. Of the 103 patients with redundant studies generated by clinicians, $73.8 \%(76 / 103)$ of the subsequent studies agreed with the first study and $26.2 \%$ (27/103) were discrepant. The difference between report discrepancy rates of RNI ordered by radiologists $(45.4 \%)$ and clinicians $(26.2 \%)$ approached significance $(P$ value $=.07)$.

The frequency of neurovascular imaging is reported in Table 2.

In 39/125 (31.2\%) patients, the report of the first and second neurovascular studies did not agree as to the presence of $\geq 50 \%$ stenosis in the head or neck. Of these 39 cases, a third study was ordered in $7(17.9 \%)$ patients and agreed with the first study in

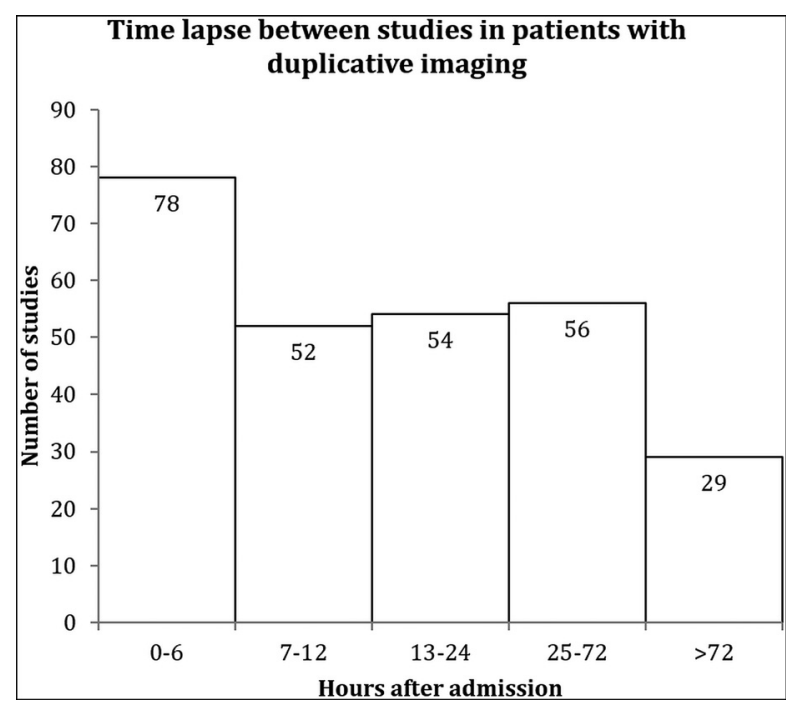

FIGURE. The time lapse between studies in patients with duplicative imaging.

Table 2: Type of neurovascular imaging ordered by clinicians and radiologists

\begin{tabular}{lc}
\hline Type of Imaging & No. (\%) \\
\hline CTA and MRA & $77(61.6)$ \\
CTA and DUS & $4(3.2)$ \\
CTA and DSA & $5(4)$ \\
MRA and DUS & $19(15.2)$ \\
MRA and DSA & $3(2.4)$ \\
CTA, MRA, DUS & $9(7.2)$ \\
CTA, MRA, DSA & $6(4.8)$ \\
MRA, CTA, CTA, DSA & $1(0.8)$ \\
MRA/CTA/MRA/DUS & $1(0.8)$ \\
Total & $125(100)$ \\
\hline
\end{tabular}

$14.3 \%$ of the cases and the second study in $85.7 \%$ of the cases. Of 86 cases in which the 2 studies agreed, a third study was ordered in $10(11.6 \%)$ cases.

Among the 125 patients with RNI, 40 (32\%) had a second imaging test after positive findings of $>50 \%$ vascular stenosis. In $85(68 \%)$ cases, the second RNI was performed after negative $(<50 \%$ stenosis) or inconclusive findings on the first imaging.

The source and discrepancy rated between modalities are reported in Table 3.

From a total of 75 MRAs and 48 CTAs performed as the first study, 51 and 33 study reports, respectively, confirmed the first study $(P$ value $=.9)$. Among all, CTA agreed with MRA, Doppler ultrasound, and DSA in 60/90 (66.7\%), 8/12 (66.7\%), and 5/9 (55.6\%) imaging studies, respectively. MRA agreed with DUS and DSA in $18 / 23(78.3 \%)$ and $4 / 6(66.7 \%)$ patients, respectively. The CTA-MRA, CTA-ultrasound, and MRA-ultrasound disagreement rates were $33.3 \%, 33.3 \%$, and $21.7 \%$, respectively.

Indications for the second study are reported in Table 4.

\section{DISCUSSION}

Our findings show that acquiring multiple vascular imaging modalities in patients diagnosed with ischemic stroke is common $(125 / 300=41.7 \%)$. Of cases of RNI, most are generated by clinicians $(82.4 \%)$, with radiologists recommending additional imaging in $17.6 \%$ of patients. What is curious is that the rate of ordering a second neurovascular study occurred 2 times more frequently (68\% versus 32\%) after a study negative for vascular stenosis than a study positive for it. Furthermore, we showed that the likelihood that the second study would be discrepant with the first study was nearly half the rate if the clinician versus the radiologist ordered it (45.4\% versus $26.2 \%$ ).

Gupta et $\mathrm{al}^{10}$ found that $58 \%$ of transferred patients with trauma underwent duplicative CT studies, with head CT primarily repeated for follow-up of trauma, and body CT primarily, because of inadequate availability or quality from the referring institution. In our series of cases, duplicative imaging studies ordered by radiologists were mostly due to uncertainty about findings and motion artifacts. Lee et $\mathrm{al}^{11}$ found that radiologists' recommendations accounted for only a small proportion (5.3\%) of outpatient CT, MR imaging, and PET examinations performed. They showed that pulmonary nodule follow-up was the most common cause of radiologist-generated high-cost imaging and was driven by pre-existing practice guidelines intended for early detection of lung cancer. ${ }^{11}$

When radiologists at our institution recommended additional studies for acute ischemic stroke evaluation, $18.2 \%$ occurred following CTA and $81.8 \%$ after MRA. This outcome may be explained by

Table 3: Source of duplicate imaging and agreement rates between modalities during the same admission

\begin{tabular}{|c|c|c|c|c|c|c|}
\hline \multirow[b]{2}{*}{$\begin{array}{l}\text { First Study } \\
\text { Ordered }\end{array}$} & \multicolumn{2}{|c|}{$\begin{array}{l}\text { Source of Second } \\
\text { Imaging }\end{array}$} & \multicolumn{3}{|c|}{ Agreement Rates between Modalities } & \multirow[b]{2}{*}{ Total } \\
\hline & Radiologist & Clinician & Agreed (\%) & $\begin{array}{c}\text { First Study Overestimated } \\
\text { Disease (\%) }\end{array}$ & $\begin{array}{c}\text { First Study Underestimated } \\
\text { Disease (\%) }\end{array}$ & \\
\hline CTA & 4 & 44 & $33(68.7)$ & $11(22.9)$ & $4(8.3)$ & 48 \\
\hline MRA & 18 & 57 & $51(68)$ & $4(5.3)$ & $20(26.7)$ & 75 \\
\hline DUS & 0 & 2 & $2(100)$ & $0(0)$ & $0(0)$ & 2 \\
\hline Total & 22 & 103 & $86(68.8)$ & $15(11.0)$ & $24(19.2)$ & 125 \\
\hline
\end{tabular}


Table 4: Indications for second study ${ }^{a}$

\begin{tabular}{lccc}
\hline Indication & Radiologist & Clinician & Total \\
\hline Patient motion & 6 & 9 & 15 \\
Technical artifact & 3 & 5 & 8 \\
Inadequate FOV & 3 & Unknown & Unknown \\
Uncertainty about findings & 7 & 4 & 11 \\
Other & 3 & Unknown & Unknown \\
\hline
\end{tabular}

${ }^{a}$ Other causes include ruling out dissection or aneurysm, follow-up, and so forth.

the higher spatial resolution, lower rate of motion artifacts, less frequent metal/air artifacts, and more reliable image quality of CTA compared with MRA. One way to reduce redundant neurovascular imaging in patients with stroke would be to use CT/CTA as the initial stroke work-up. Although there was a propensity for the second study to confirm the findings of the first study (68.8\%), the $31.2 \%$ rate of discordance suggests that the reason for ordering a corroborative RNI may have been justified. It reversed the initial finding in nearly one-third of cases. By contrast, the clinicians' RNIs showed a reversal rate of nearly half that of the radiologists $(26.2 \%$ versus $45.4 \%)$.

Increased imaging use could be due to the increased availability and capacity of scanners during the past decades. While imaging use represents a growing proportion of health care expenditures, the influence of radiologists' self-referral on high-cost imaging volume is small. Hence, efforts to alter the radiologist's behavioral patterns in making recommendations for additional studies are unlikely to result in a substantial decrease in national health care costs. Additionally, based on a study by Sistrom et al, ${ }^{12}$ the odds of radiologists making a recommendation for additional imaging in their reports decreased with experience by approximately $15 \%$ per decade.

Another study from Lee et $\mathrm{al}^{13}$ showed that self-referral by a radiologist through recommendations for redundant imaging in examination reports contributes very little to the overall volume of high-cost imaging; only $8 \%$ of studies are performed after a radiologist's recommendation for a repeat study (compared with our 22/300 [7.3\%] rate for stroke patients). In their study, certain examinations with relatively higher repeat rates, such as chest CT (15\%) and pelvic ultrasound (8\%), represent specific situations in which imaging follow-up is one of the recognized methods for patient care. The authors recommended defining scenarios in which high-cost imaging studies have the maximal medical effect and developing consensus multidisciplinary recommendations for high-cost examination use in those clinical situations. ${ }^{13}$

\section{Limitations of Our Study}

Our study has some limitations that impact its conclusions. This study was a single-institution review with a modest sample size and is limited to the evaluation of neurovascular imaging modalities chosen because of the multiple modalities (CTA, MRA, DUS, and DSA) available for clinicians to order. The other limitation of our study was that we cannot always assess from the medical record the rationale, in a retrospective study, of clinicians when they order redundant modalities and we cannot reconstruct conversations that may have occurred between health care providers that may have led to ordering studies. Additionally, among our patient sample, we had some combinations of modalities (ie, DSA/DUS) with so few incidents that we cannot make conclusions about accuracy rates. We also understand that there is some blurring of distinction between redundant imaging (implying little potential added value) versus repeat imaging (because of a changing clinical situation). Certain scenarios warrant close follow-up neurovascular imaging such as the following: 1) a patient who comes in with acute stroke due to dissection or nonocclusive intravascular thrombus who has new symptoms suggestive of an acute occlusion that might be treatable with thrombectomy; 2) a patient with dissection in whom a vessel is suggested to be occluded on 1 technique but confirmation is requested before sending the patient home on aspirin instead of anticoagulation; and 3) the patient with borderline NASCET criteria with fluctuating neurologic examination findings. Finally, our study is impacted by our institutional bias in favor of MR imaging/MRA for acute stroke evaluation and the availability for scheduling these emergently, given that we have an MR imaging scanner in our emergency department. This scenario may differ with the practice patterns of other radiology groups. We recommend a global assessment of the impact of RNI, which can lead to recommended practice parameters by national organizations.

\section{Implications/Recommendations}

During hand-offs between services, miscommunication can occur, leading to potential redundancies. One wonders whether there would be value in electronic reminders to clinicians that a previous neurovascular study had been performed, as part of the orderentry process of the physician. This would be an easy radiology information system/EMR (Electronic Medical Record) intervention. Such a feedback loop alerting the ordering physician ("You are aware that the patient had a DUS earlier today") may help avoid inadvertent, unintended RNIs. If radiologists who are unsure consult with colleagues before recommending additional tests due to uncertainty about findings, it may reduce RNIs. If clinicians discuss their concerns about studies with radiologists before ordering another technique, it may decrease redundant imaging. In the end, communication among practitioners may help address these problems.

\section{CONCLUSIONS}

Our hypothesis that radiologists would account for $<10 \%$ of redundant neurovascular studies in the setting of stroke was incorrect. Radiologists recommended another technique in $17.6 \%$ of patients with RNI and $7.3 \%$ of all patients with stroke. The concordance rate of the 2 RNI modalities was much higher when those studies were clinician-driven; therefore, it implies limited second-study benefit. Such redundancy should be discouraged. Duplicative neurovascular studies on the same patient occur frequently, often agree, and are a source of added cost. Because clinicians and radiologists contribute to RNI, both specialties should be circumspect in their ordering habits. RNI should be addressed at a national level to reduce health care costs, and its use could benefit from order-entry feedback loops.

Disclosures: David M. Yousem—UNRELATED: Expert Testimony: expert witness testimony; Payment for Lectures Including Service on Speakers Bureaus: American College of Radiology Education Center speaker, mrionline.com; Royalties: Elsevier for 5 books, Analytical Informatics. 


\section{REFERENCES}

1. Nederkoorn PJ, van der Graaf Y, Hunink M. Duplex ultrasound and magnetic resonance angiography compared with digital subtraction angiography in carotid artery stenosis: a systematic review. Stroke 2003;34:1324-32 CrossRef Medline

2. Hassan AE, Rostambeigi N, Chaudhry SA, et al. Combination of noninvasive neurovascular imaging modalities in stroke patients: patterns of use and impact on need for digital subtraction angiography. J Stroke Cerebrovasc Dis 2013;22:e53-58 CrossRef Medline

3. Adla T, Adlova R. Multimodality imaging of carotid stenosis. Int $J$ Angiol 2015;24:179-84 CrossRef Medline

4. Barnett HJ, Taylor DW, Haynes RB, et al; North American Symptomatic Carotid Endarterectomy Trial Collaborators. Beneficial effect of carotid endarterectomy in symptomatic patients with high-grade carotid stenosis. N Engl J Med 1991;325:445-53 CrossRef Medline

5. Papanicolas I, Woskie LR, Jha AK. Health care spending in the United States and other high-income countries. JAMA 2018;319:1024-39 CrossRef Medline

6. Haley T, Ghaemmaghami V, Loftus T, et al. Trauma: the impact of repeat imaging. Am J Surg 2009;198:858-62 CrossRef Medline
7. Prabhakar AM, Gottumukkala RV, Hemingway J, et al. Increasing utilization of emergency department neuroimaging in Medicare beneficiaries from 1994 to 2015. Am J Emerg Med 2018;36:680-83 CrossRef Medline

8. Levine DA, Burke JF. Stroke imaging: quantity, but is there quality? Med Care 2016;54:423-25 CrossRef Medline

9. Adams HP, Bendixen BH, Kappelle LJ, et al. Classification of subtype of acute ischemic stroke. Definitions for use in a multicenter clinical trial. TOAST. Trial of Org 10172 in Acute Stroke Treatment. Stroke 1993;24:35-41 CrossRef Medline

10. Gupta R, Greer SE, Martin ED. Inefficiencies in a rural trauma system: the burden of repeat imaging in interfacility transfers. $J$ Trauma 2010;69:253-55 CrossRef Medline

11. Lee SI, Krishnaraj A, Chatterji M, et al. When does a radiologist's recommendation for follow-up result in high-cost imaging? Radiology 2012;262:544-49 CrossRef Medline

12. Sistrom CL, Dreyer KJ, Dang PP, et al. Recommendations for additional imaging in radiology reports: multifactorial analysis of 5.9 million examinations. Radiology 2009;253:453-61 CrossRef Medline

13. Lee SI, Saokar A, Dreyer KJ, et al. Does radiologist recommendation for follow-up with the same imaging modality contribute substantially to high-cost imaging volume?. Radiology 2007;242:857-64 CrossRef Medline 